\title{
LETTER \\ Supporting Jogging at an Even Pace by Synchronizing Music Playback Speed with Runner's Pace*
}

\author{
Tetsuro KITAHARA $^{\dagger a)}$, Member, Shunsuke HOKARI ${ }^{\dagger}$, and Tatsuya NAGAYASU ${ }^{\dagger}$, Nonmembers
}

\begin{abstract}
SUMMARY In this paper, we propose a jogging support system that plays back background music while synchronizing its tempo with the user's jogging pace. Keeping an even pace is important in jogging but it is not easy due to tiredness. Our system indicates the variation of the runner's pace by changing the playback speed of music according to the user's pace variation. Because this requires the runner to keep an even pace in order to enjoy the music at its normal speed, the runner will be spontaneously influenced to keep an even pace. Experimental results show that our system reduced the variation of jogging pace.

key words: jogging, healthcare, music, smartphone
\end{abstract}

\section{Introduction}

Computer-aided prevention of disease and/or disorders has recently attracted a good deal with attention, and accordingly computing technologies such as those for promoting healthy behavior are expected to bring beneficial effects [3]. One of the disorders to prevent is the metabolic syndrome. To prevent the metabolic syndrome, increasing physical activity is important. A simple but effective way for increasing physical activity is jogging. The importance of jogging is in maintaining an even pace from beginning to end [4], but this may not be easy for novices. Novice runners often decrease their pace due to tiredness. If computing technologies can alert these runners of their pace variations to help them keep an even pace, it would be useful in allowing them to enjoy jogging.

We focus on the fact that a number of people jog while listening to music with their portable music players (including smartphones). We then propose a method for telling the runner the pace variation by synchronizing the music playback speed with the runner's pace. After the runner's standard pace is measured, the playback speed automatically increases or decreases, according to the runner's pace as it increases or decreases. This method requires the runner to keep the standard pace in order to enjoy the music at its normal speed, therefore we expect that this method will raise his/her awareness of pace control and spontaneously influence him/her to keep an even pace.

There have been many attempts to support jogging with

Manuscript received September 30, 2014.

Manuscript revised December 11, 2014.

Manuscript publicized January 9, 2015.

$\dagger$ The authors are with College of Humanities and Sciences, Nihon University, Tokyo, 156-8550 Japan.

*This paper is an extended version of the papers presented at IPSJ National Convention 2013 [1] and HCI International 2014 [2].

a)E-mail: kitahara@chs.nihon-u.ac.jp

DOI: 10.1587/transinf.2014EDL8199 computing technologies (e.g., [5], [6]) and some of them have focused on music [7]-[12]. Rubisch et al. [7] developed a mobile-phone-based system that recommends musical pieces that have a close tempo to the target heart rate defined by the user-desired amount of oxygen consumption. Oliver et al. [8] also developed a mobile-phone-based system, called MPTrain, which measures the user's physiological condition such as the heart rate and selects musical pieces that will encourage the user to speed up, slow down, or keep the pace. Nirjon et al. [9] and Adachi et al. [10] also developed similar systems. These systems use the heart rate as a measure of the user's condition, but the measurement of the heart rate requires a dedicated device (no music players or smartphones have a built-in function of measuring the heart rate). These systems adopt the approach of selecting musical pieces that have an appropriate tempo rather than changing the tempo of the musical piece being played back.

Ohira et al. [11] developed a music player adapted to the walking or jogging rhythm. This system uses an acceleration sensor for measuring the rhythm of walking/jogging instead of the heart rate. The advantage of this system is that it does not require a dedicated device, because most recent mobile devices have an acceleration sensor. Sakata et al. [12] also proposed a similar concept called interactive jogging. However, the effectiveness of these systems has not been confirmed through experiments.

In this paper, we propose a music player that has the following features:

- The user's behavior is measured only by the acceleration sensor and does not require any dedicated devices.

- The variation of the user's pace is immediately reflected in the playback speed of the musical piece being played back to allow the user to be aware of his/her pace variation in real time.

\section{Proposed System}

The user wears an Android device on his/her hip using a belt and jogs while listening to music using the earphones or headphones connected to the Android device. After the music starts, it is then played back at the normal speed for 15 seconds, because the first five seconds are regarded as a setup time for running and the next ten seconds are used for measuring the runner's standard pace. After measuring the standard pace, the system starts to synchronize the playback speed with the user's pace based on the following method. 


\subsection{Preparation of Audio Signals}

Audio signals of the target music with different playback speeds are generated on a PC in advance. In the current implementation, those with $0.5,0.6, \ldots, 2.0$ times of the original speed are generated based on Phase Vocoder [13] using the MARY Text To Speech Libary [14].

\subsection{Measurement of Jogging Pace}

The pace is measured with the acceleration sensor built in the Android device. The acceleration value $\alpha(t)$ is defined by:

$$
\begin{aligned}
\alpha(t)= & \left|a_{x}(t)-a_{x}(t-1)\right|+\left|a_{y}(t)-a_{y}(t-1)\right| \\
& +\left|a_{z}(t)-a_{z}(t-1)\right|
\end{aligned}
$$

and is measured every $80 \mathrm{~ms}$, where $a_{x}(t), a_{y}(t)$, and $a_{z}(t)$ are the accelerations along the $\mathrm{x}_{-}, \mathrm{y}-$, and $\mathrm{z}$-axes, respectively. When $\alpha(t)$ is higher than an experimentally determined threshold $\alpha_{\theta}$, the step counter is incremented. The number of steps for the previous four seconds is regarded as the jogging pace at that time.

The previous methods [11], [12] use the acceleration only in the vertical direction. However, the relationship between the sensor's internal axes and the external axes (i.e., which of the $\mathrm{x}_{-}, \mathrm{y}-$, and $\mathrm{z}$-axes is vertical) depend on how the user wares the device. In jogging, the non-vertical accelations are expected to be sufficiently smaller than the vertical accelation. We therefore simply add all of the three directional accelations.

\subsection{Switching of Playback Speed}

For every second, the system calculates the pace and accordingly switches the audio signal. As an example, when the pace at time $t$ is 0.8 times the standard pace, the audio signal with 0.8 times of the normal speed is used.

\section{Experiments}

\subsection{Experimental Condition}

We conducted experiments on jogging using our system. There were ten participants, ages 22 to 23. Participant E exercises every month and Participant $\mathrm{H}$ exercises more than three times a week, while the remaining eight partcipants usually do not exercise. The procedure was as follows:

1. Try a two-minute jog three times, listening to music with the normal playback speed, at 30-s intervals.

2. Rest for enough time.

3. Try a two-minute jog three times again, listening to music with our system, at 30-s intervals.

To avoid order effects, we replaced Step 1 with Step 3 for half of the participants.
We used a straight course and asked the participants to turn back around one minute after they started. In choosing a musical piece, we should prevent that the results are affected by whether the participants know the piece used. We therefore chose a musical piece from RWC Music Database [15], no pieces in which were known to any participants. From this database, we took "Tonight's the Night for Love" because it is suited to background music of jogging ${ }^{\dagger}$. As an Android device, we used an Acer ICONIA TAB A100 with Android 3.2.1.

\subsection{Experimental Results}

Table 1 lists the temporal average and coefficients of variation of the every-second jogging pace for each trial. Unfortunately the data for Participants I and J were lost due to device trouble, and those were removed from the table. The results are summarized as follows:

1. Participant A's pace decreased at every trial with normal speed playback. Participant B's pace also varied between the trials. On the other hand, they jogged at consistent paces with our system.

2. The coefficients of variation for the 2 nd and 3 rd trials were smaller with our system than with normal speed playback except for the 3rd trials by Participants E and $\mathrm{G}$. This means that our system reduced pace variation caused by tiredness to some extent. For the 3rd trial by Participant E, the coefficient of variation with our system was larger than that with the normal speed playback. This may be because he frequently exercises. For the 3rd trial by Participant G, the coefficients of variation with our system was approximately equal to that with the normal speed playback.

Table 1 Temporal average and coefficients of variation of pace for each trial.

\begin{tabular}{cllllll}
\multicolumn{8}{c}{ (a) Our System } \\
\hline Participant & \multicolumn{2}{c}{ Trial 1 } & \multicolumn{2}{c}{ Trial 2 } & \multicolumn{2}{c}{ Trial 3 } \\
\hline A & 28.0 & 0.071 & 21.0 & 0.095 & 21.0 & 0.14 \\
B & 21.5 & 0.19 & 23.8 & 0.16 & 22.4 & 0.13 \\
C & 22.8 & 0.18 & 22.4 & 0.16 & 21.4 & 0.16 \\
D & 27.5 & 0.12 & 30.0 & 0.12 & 29.6 & 0.11 \\
E & 30.2 & 0.17 & 27.7 & 0.14 & 28.8 & 0.17 \\
F & 11.1 & 0.18 & 12.0 & 0.19 & 11.2 & 0.16 \\
G & 27.0 & 0.15 & 22.8 & 0.15 & 19.4 & 0.17 \\
H & 24.7 & 0.15 & 29.5 & 0.17 & 30.1 & 0.15 \\
\hline
\end{tabular}

\begin{tabular}{crlllll}
\multicolumn{7}{c}{ (b) Normal Speed Playback } \\
\hline Participant & \multicolumn{2}{c}{ Trial 1 } & \multicolumn{2}{c}{ Trial 2 } & \multicolumn{2}{c}{ Trial 3 } \\
\hline A & 29.5 & 0.017 & 22.5 & 0.47 & 15.5 & 0.22 \\
B & 10.4 & 0.27 & 20.9 & 0.25 & 24.2 & 0.15 \\
C & 26.4 & 0.16 & 23.2 & 0.19 & 23.3 & 0.18 \\
D & 26.2 & 0.13 & 26.3 & 0.14 & 25.7 & 0.14 \\
E & 27.2 & 0.19 & 26.1 & 0.16 & 27.4 & 0.15 \\
F & 10.8 & 0.15 & 12.5 & 0.30 & 12.7 & 0.18 \\
G & 19.3 & 0.20 & 19.4 & 0.21 & 18.6 & 0.17 \\
H & 8.2 & 0.26 & 21.8 & 0.18 & 24.6 & 0.20 \\
\hline
\end{tabular}

This is a newly composed piece for this database, and therefore different from the piece with the same title by Al Johnson. 

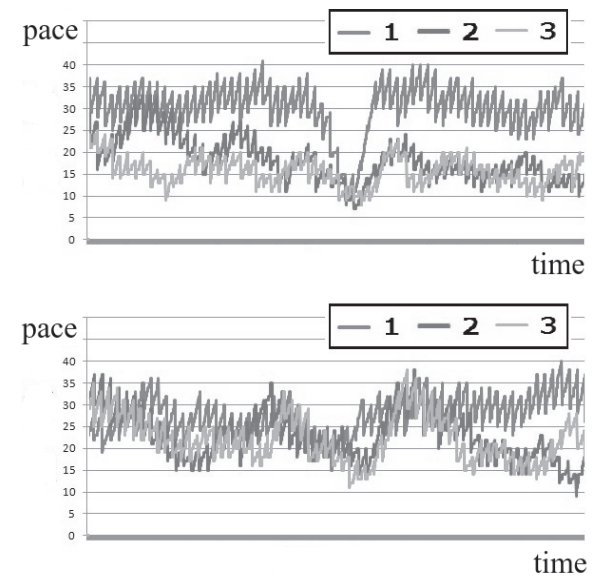

Fig. 1 Pace by Participant A with normal playback (upper) and with our system (lower).

Table 2 Results of questionnaire.

\begin{tabular}{c|cccc}
\hline Participant & Q1 & Q2 & Q3 & Q4 \\
\hline A & 2 & 5 & 5 & 5 \\
B & 4 & 4 & 4 & 5 \\
C & 4 & 6 & 6 & 3 \\
D & 6 & 7 & 4 & 6 \\
E & 1 & 4 & 2 & 4 \\
F & 3 & 7 & 6 & 5 \\
G & 4 & 7 & 6 & 5 \\
H & 3 & 6 & 6 & 6 \\
I & 3 & 7 & 4 & 7 \\
J & 3 & 6 & 5 & 6 \\
\hline Avg. & 3.3 & 5.9 & 4.8 & 5.2 \\
\hline
\end{tabular}

Details of every-second paces by Participant A are shown in Fig. 1. The pace was reduced for each trial with normal speed playback, whereas the pace with our system was not reduced. The pace largely decreased on turning back for normal speed playback, while the pace did not decrease with our system. This would be an effect of synchronizing music playback with the running pace.

\subsection{Questionnaire}

We asked the ten participants to answer a questionnaire consisting of the following questions:

Q1 Did the music playback synchronized to your pace?

Q2 Did you consciously control the jogging pace according to the music?

Q3 Did you feel that jogging becomes easy with our system?

Q4 Do you want to use this system in your daily life?

The questionnaire was conducted on a scale of one to seven.

The results are shown in Table 2. The result for Q1 was not high. Our system updates the playback speed at every second according to the average pace during last four seconds, so a little latency occurred in the synchronization. This is why the result of Q1 was not high. The result for Q2 was high. It means that our approach successfully raised the runner's consciousness of pace control. The results for Q3
Table 3 Participants' comments (excerpt).

The change of the playback speed was more frequent than expected. It could be better to keep the same playback speed at least two seconds. I would like to try this system with other musical pieces. I'm interested in what happens when I go up the stairs.

It was interesting. The playback sufficiently closely followed my running pace although there was a little bit latency. The sound quality was also good. The change of playback speed was good because it's not insistent, compared to indication by voice such as "too fast" and "too slow."

Some people may want to enjoy music independently of jogging. This system is not suitable for such people. The margin of the pace for the normal playback speed should be a litte more broader.

It will be interesting if this is released as a smartphone app. The sound quality was no problem. I felt latency. I tried to jog according to the tempo of the music. It made the jogging easy.

The synchronization had latency. However, it was interesting that I can recognize my pace of running.

I took care to keep the speed of music during the running. Due to it, I didn't feel very much pain. It was interesting.

and Q4 were also high on average. This means that our system is useful to support jogging by people without frequent exercise.

Table 3 lists an excerpt of the comments given by the participants. Except for the latency of synchronization, they had positive impression on the whole.

\section{Conclusion}

To encourage the runner to maintain an even pace in jogging, we developed a system for indicating pace variation by synchronizing the music playback speed with the running pace. Experimental results showed that this system raised the runner's awareness of pace control and successfully reduced pace variation.

In the current implementation, it is necessary to prepare audio signals with various playback speeds in advance on a PC. To solve this problem, we plan to implement the Phase Vocoder [13] and real-time music synchronization on the Android platform. We also plan to distribute this software to collect users' feedback for further improvement.

\section{Acknowledgments}

This work is supported by JSPS KAKENHI Grant Number 26240025.

\section{References}

[1] S. Hokari, T. Nagayasu, and T. Kitahara, "Music player for smartphone synchronizing playback speed with jogging pace," Proc. IPSJ National Convention, vol.3, pp.249-250, 4W-4, 2013.

[2] T. Kitahara, S. Hokari, and T. Nagayasu, "Music synchronizer with runner's pace for supporting steady pace jogging," HCI International 2014 - Posters' Extended Abstracts, Communications in Computer and Information Science, pp.343-348, Springer, 2014.

[3] C. Yum, "The use of personalized, mobile applications in preventative healthcare," http://sydney.edu.au/engineering/it/ judy/Teach/ Reading09/04_Yum.pdf

[4] B. Cooper, "The 25 golden rules of running," http://www.runnersworld.com/running-tips/25-golden-rules-running 
[5] M. Makikawa, T. Isaka, T. Iida, S. Asajima, K. Tsukise, N. Shiozawa, K. Nishiyama, S. Wada, K. Nakayama, M. Horiguchi, and H. Torimoto, "Portable jogging monitor device and its application for health management," Proc. APBME, pp.40-41, 2003.

[6] F.F. Mueller, S. O'Brien, and A. Throgood, "Jogging over a distance: Supporting a "jogging together" experience although being apart," Proc. CHI, pp.2579-2584, 2007.

[7] J. Rubisch, M. Husinsky, J. Doppler, H. Rffaseder, B. Horsak, B. Ambichi, and A. Figl, "A mobile music concept as support for achieving target heart rate in preventive and recreational endurance training," Proc. Audio Mostly Conf., Article no.19, 2010.

[8] N. Oliver and F. Flores-Mangas, "MPTrain: A mobile, music and physiology-based personal trainer," Proc. MobileHCI, pp.21-28, 2006.

[9] S. Nirjon, R.F. Dickerson, Q. Li, P. Asare, and J.A. Stankovic, "MusicalHeart: A heaty way of listening to music," Proc. SenSys, pp.43-56, 2012.

[10] K. Adachi and Y. Nakatani, "System that supports change of exercise tempo in sports by the music rhythm," Proc. 3rd Asian Workshop on Inf. Tech., p.78, 2010.

[11] S. Ohira and K. Nagao, "Music player system adapted to walking/jogging rhythm,” Proc. JSAI 2007, 3C7-3, 2007 (in Japanese).

[12] N. Sakata, T. Kurata, M. Kourogi, and H. Kuzuoka, "Situated music: An application to interactive jogging," Proc. ISWC, Student Colloquium, 2006.

[13] J.L. Flanagan and R.M. Golden, "Phase vocoder," Bell Syst. Tech. J., vol.45, pp.1492-1509, 1966.

[14] “Mary text to speech," http://mary.dfki.de/

[15] M. Goto, H. Hashiguchi, T. Nishimura, and R. Oka, "RWC music database: Music genre database and musical instrument sound database," Proc. ISMIR, pp.229-230, 2003. 